Supporting Information

\title{
Environmental Mercury Chemistry - In Silico
}

\section{Abu Asaduzzaman, ${ }^{1,2}$ Demian Riccardi, ${ }^{3,4}$ Akef T. Afaneh, ${ }^{1,5}$ \\ Connor J. Cooper, ${ }^{6}$ Jeremy C. Smith, ${ }^{3,4}$ Feiyue Wang, ${ }^{7}$ Jerry M. Parks, ${ }^{3,6}$ and Georg Schreckenbach ${ }^{{ }^{*}}$}

1 Department of Chemistry, University of Manitoba, Winnipeg, MB, Canada, R3T 2N2

2 School of Science, Engineering and Technology, Penn State Harrisburg, 777 West Harrisburg Pike, Middletown, PA 17057, USA

3 University of Tennessee/Oak Ridge National Laboratory Center for Molecular Biophysics, Biosciences Division, Oak Ridge National Laboratory, 1 Bethel Valley Road, Oak Ridge, TN 37831-6309, USA

4 Department of Biochemistry and Cellular and Molecular Biology, University of Tennessee Knoxville, Knoxville, Tennessee 37996, USA

5 Department of Chemistry, Faculty of Science, Al-Balqa Applied University, P. O. Box 19117, postal code 19117, Al-Salt, Jordan

6 Graduate School of Genome Science and Technology, University of Tennessee, Knoxville, Tennessee 37996, USA

7 Centre for Earth Observation Science and Department of Environment and Geography, University of Manitoba, Winnipeg, MB, Canada, R3T 2N2

*schrecke@cc.umanitoba.ca 
Table S1. Mulliken charges (in electron) on $\mathrm{Hg}, \mathrm{S} / \mathrm{Se}$ and $\mathrm{N}$ atoms and HOMO and LUMO energies (in $\mathrm{eV}$ ) of $\mathrm{MeHg}$ amino acid and selenoamino acid complexes. ${ }^{51}$

\begin{tabular}{lccccc}
\hline & \multicolumn{3}{c}{ Mulliken charge } & \multicolumn{2}{c}{ Energy } \\
\cline { 2 - 6 } Complexes & $\mathrm{Hg}$ & $\mathrm{S} / \mathrm{Se}$ & $\mathrm{N}$ & HOMO & LUMO \\
\hline MeHgCys & 0.51 & -0.10 & -6.17 & -0.89 \\
MeHgSeCys & 0.61 & -0.27 & -5.94 & -0.96 \\
MeHgPen & 0.61 & -0.40 & -6.16 & -0.84 \\
MeHgSePen & 0.63 & -0.32 & -5.89 & -0.88 \\
MeHgGlu & 0.63 & -0.13 & -6.34 & -1.00 \\
MeHgSeGlu & 0.72 & -0.32 & -6.09 & -1.05 \\
MeHgMeth_lowpH ${ }^{\mathrm{a}}$ & 0.51 & 0.92 & 0.55 & -14.25 & -8.32 \\
MeHgSeMeth_lowpH & 0.67 & 0.36 & 0.55 & -13.94 & -8.33 \\
MeHgMeth_highpH $^{\mathrm{a}}$ & 0.84 & 0.09 & & -6.03 & -1.22 \\
MeHgSeMeth_highpH $^{\mathrm{a}}$ & 0.84 & 0.06 & & -5.75 & -1.23 \\
\hline
\end{tabular}

a ... "lowpH" and "highpH" refer to different binding modes of methylmercury as models of different $\mathrm{pH}$ conditions; see below and ref. 51 for details. 
Table S2. Formation reactions of $\mathrm{Hg}$ complexes; various energies. ${ }^{51}$ This table is an extended version of Table 1; a discussion of the various energies is given below. In Table 1, $\Delta \mathrm{E}_{\text {water }}^{S}$ has been reported as $\Delta \mathrm{G}$. Values in $\mathrm{kcal} / \mathrm{mol}$.

\begin{tabular}{lrrr}
\hline Reaction & \multicolumn{1}{c}{$\Delta \mathbf{G}_{\text {gas }}$} & \multicolumn{1}{c}{$\Delta \mathbf{G}_{\text {water }}$} & \multicolumn{1}{c}{$\Delta \mathrm{E}_{\text {water }}^{S}$} \\
\hline Reaction (S1), E = S & -8.4 & -6.4 & -5.7 \\
Reaction (S1), E = Se & -14.8 & -11.4 & -10.6 \\
\hline Reaction (2): Reagent AH =? & & & \\
\hline cysteine & -18.1 & -16.8 \\
selenocysteine & -22.2 & -19.0 \\
penicillamine & -14.8 & -12.1 \\
selenopenicillamine & -22.6 & -19.9 \\
gluthathione & -18.3 & -14.6 \\
selenogluthathione & -22.7 & -18.8 \\
methionine (low pH model) & -29.9 & 7.6 \\
selenomethionine (low pH model) & -32.08 & 7.0 \\
methionine (high pH model) & 1.8 & 6.7 \\
selenomethionine (high pH model) & 1.3 & 2.3 \\
\hline
\end{tabular}

\section{Discussion of Table S2:}

The following discussion has been adapted from Ref. 51. It illustrates, by way of example, details of the process involved in building an appropriate model for comparing methylmercury complexes of amino acids and their selenoamino acid counterparts. In addition, it provides the details for the different types of energies reported in the Table. 
Complex formation reactions using $\mathrm{CH}_{3} \mathrm{HgOH}$ as the $\mathrm{Hg}$ reagent were chosen as a model for the in vivo thermodynamic competition between the $\mathrm{S}$ and Se complexes. The first two rows in Table S1 concern formation reactions of $\mathrm{CH}_{3} \mathrm{HgECH}_{3}, \mathrm{E}=\mathrm{S}$ and Se.

$$
\mathrm{CH}_{3} \mathrm{HgOH}+\left(\mathrm{CH}_{3}\right)_{2} \mathrm{E} \rightarrow \mathrm{CH}_{3} \mathrm{HgECH} \mathrm{H}_{3}+\mathrm{CH}_{3} \mathrm{OH}
$$

These reactions serve as a model for compounds containing $\mathrm{CH}_{3} \mathrm{Hg}-\mathrm{E}$ bonds. Within the current context, the most important difference is that between the free energy in solution, $\Delta \mathrm{G}_{\mathrm{water}}$ and a variant thereof, $\Delta \mathrm{E}_{\text {water }}^{S}$. Both are free energies in solution that were calculated with continuum solvation (the CPCM method in this case ${ }^{51}$ ). However, $\Delta \mathrm{G}_{\mathrm{water}}$ was calculated from full optimization of reactants and products in solution, whereas $\Delta \mathrm{E}_{\text {water }}^{S}$ was obtained from singlepoint solution calculations based on gas-phase geometries. We note a systematic error of about 1 $\mathrm{kcal} / \mathrm{mol}$ in $\Delta \mathrm{E}_{\text {water }}^{S}$ compared to $\Delta \mathrm{G}_{\text {water }}$ (Table S2). In our study, we have assumed transferability of this result to the larger (seleno)amino acid complexes, and hence we report $\Delta \mathrm{E}_{\text {water }}^{S}$ data from these (as $\Delta \mathrm{G}$ in Table 1).

Methionine and its selenoamino acid analogue provide a special case. Its binding to methylmercury depends on $\mathrm{pH}$. At low $\mathrm{pH}, \mathrm{Hg}$ binds to the $-\mathrm{SH}(-\mathrm{SeH})$ group, as is the case for the other amino acids. However, at higher $\mathrm{pH}$, binding at the amino group occurs instead. Both forms have been considered, see Table $\mathbf{S 2}$ and the original paper; only the "high-pH" data has been included in Table 1. 